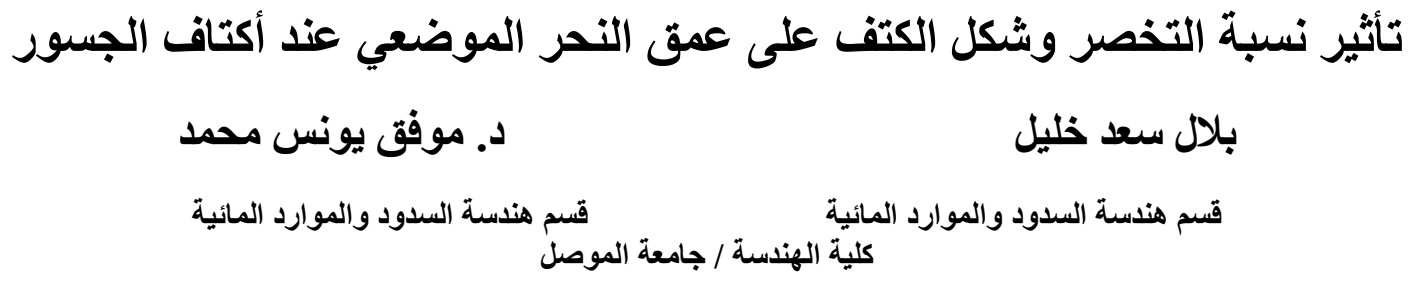

\title{
الخلاصة
}

تتأثر عملية النحر عند أكتاف الجسور بتقلص مجرى الماء خصوصا عندما تثثل الأكتاف حيزا مؤثر من مسار

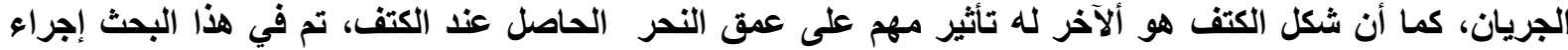

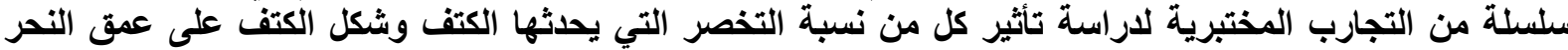

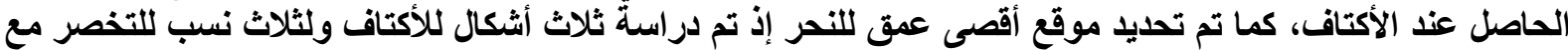

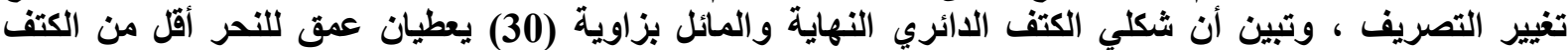

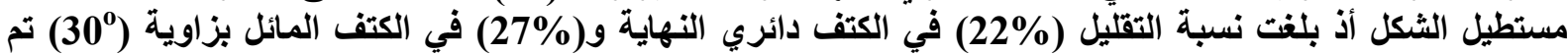

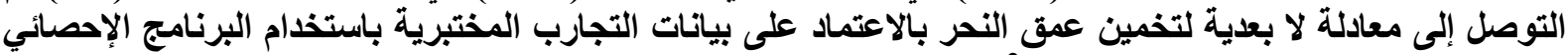
(SPSS 11.5) الكلمات اللالة: النحر، الأكتاف، التخصر.

\section{Effect of the Contraction ratio and the Shape of the Abutment on the Local Scour at a Bridge Abutments}

\author{
Bilal. S K* \\ Dr. Mwafaq. Y M**
}

\begin{abstract}
Scour process at the abutment of bridge is influenced by the contraction of the stream flow specially when the abutment immure an effective width of the stream flow, also the shape of the abutment has an important effects on the scour depth at the abutment. In this paper a series of laboratory experiments were carried out to study the effect of contraction ratio and abutment shape on the scour and its maximum depth at the abutment. Three shapes of abutments with three-contraction ratio with different flow rates were used. The result of the experiments showed that the circular shape and $\left(30^{\circ}\right)$ angle abutments reduce scour compared with that, which was, occur at the rectangular abutment. The reduction of scour depth with circular shape is about $(22 \%)$ where that with $\left(30^{\circ}\right)$ angle is about $(27 \%)$, also a dimensionless general empirical formula was created for the calculation the scour depth by using the (SPSS 11.5) Statistical program a Coefficient $\left(R^{2}=0.933\right)$ of determination.
\end{abstract}

Keywords: Scour, Abutment, Contraction. 


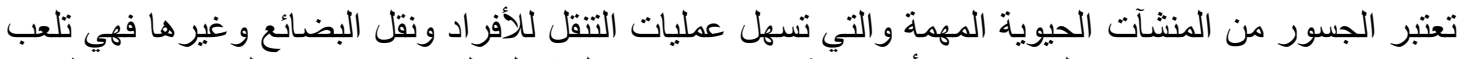

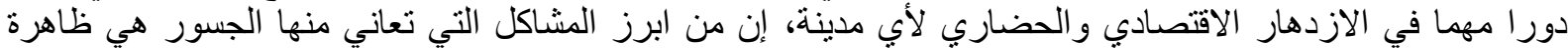

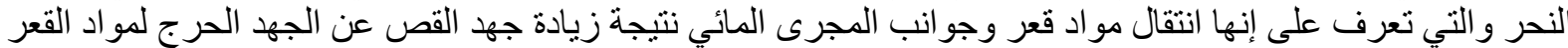

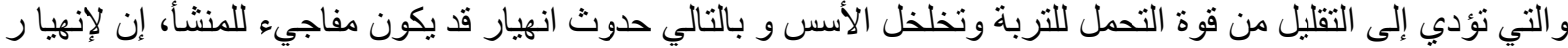

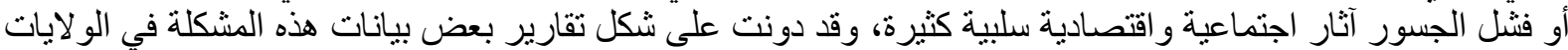

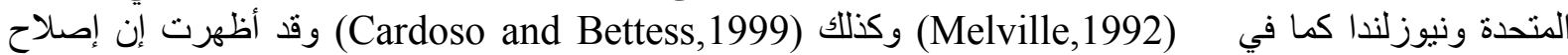

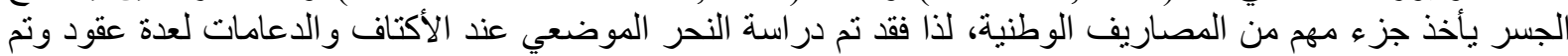

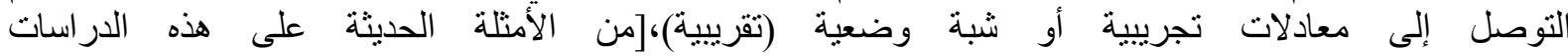

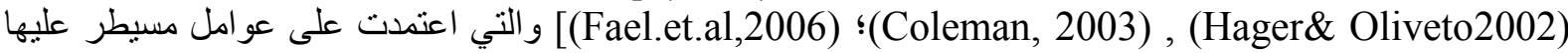
لإيجاد عمق النحر وذللك بتمثيل النظام بصيغة لا بعديه. ودرس باليو وآخرون (Ballio et.al, 2009) النحر الموضعي نتيجة التخصرات لتحديد تأثثير التقلص في مسار الجريان على النئل النحر الوضعي، وأجرى يوسفبور وآخرون (Yousefpour. et. al, 2011) الثبكات العصبية الصناعية.

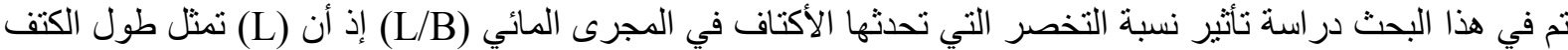

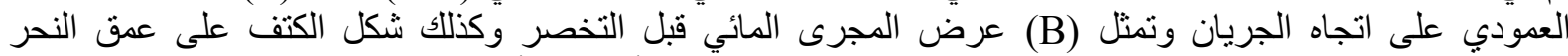

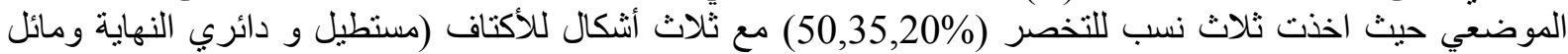

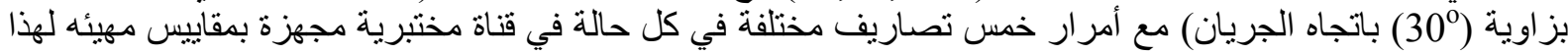

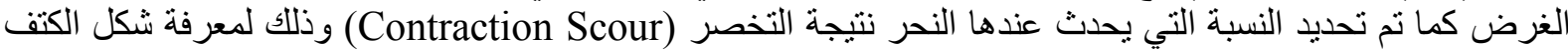
الذي يعطي أقل عمق للنحر وتم التوصل إلى معادلة لا بعدية لتمثيل عملية النحر عند كل شكل من أثنكال الأكتاف تحت

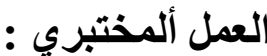

أجريت جميع تجارب هذا البحث في مختبر الهيدروليك التابع لقسم هندسة السدود و الموارد المائية - جامعة

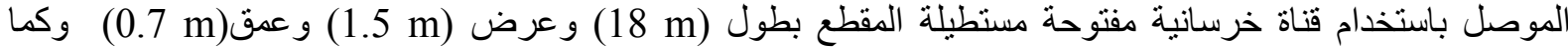

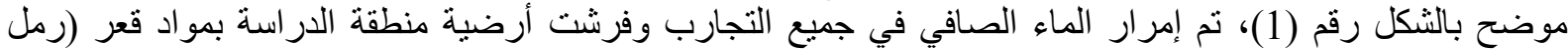

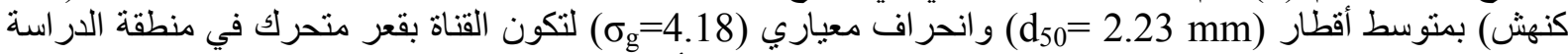

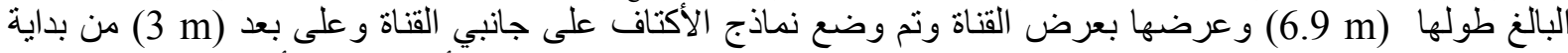

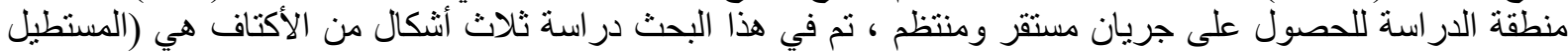

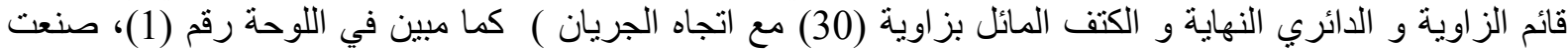

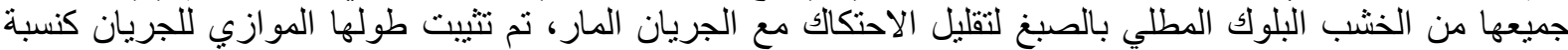

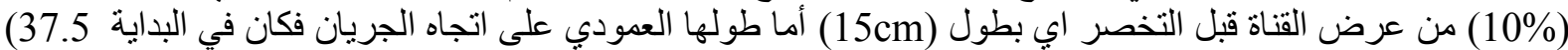

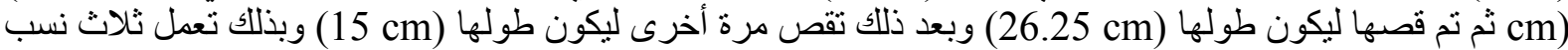

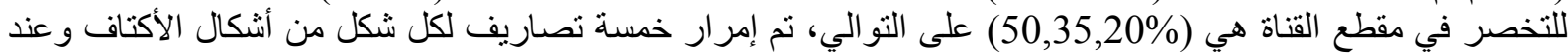

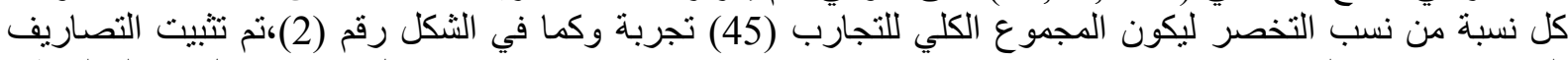

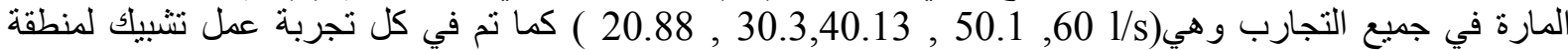

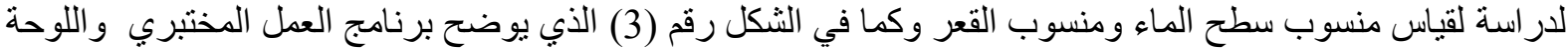
(2) نموذج للنحر الحاصل عندالكثف دائري النهاية ولنسبة تخصر (50\%). 
محمد: تأثير نسبة التخصر وشكل الكتف على عمق النحر الموضعي عند أكتاف الجسور

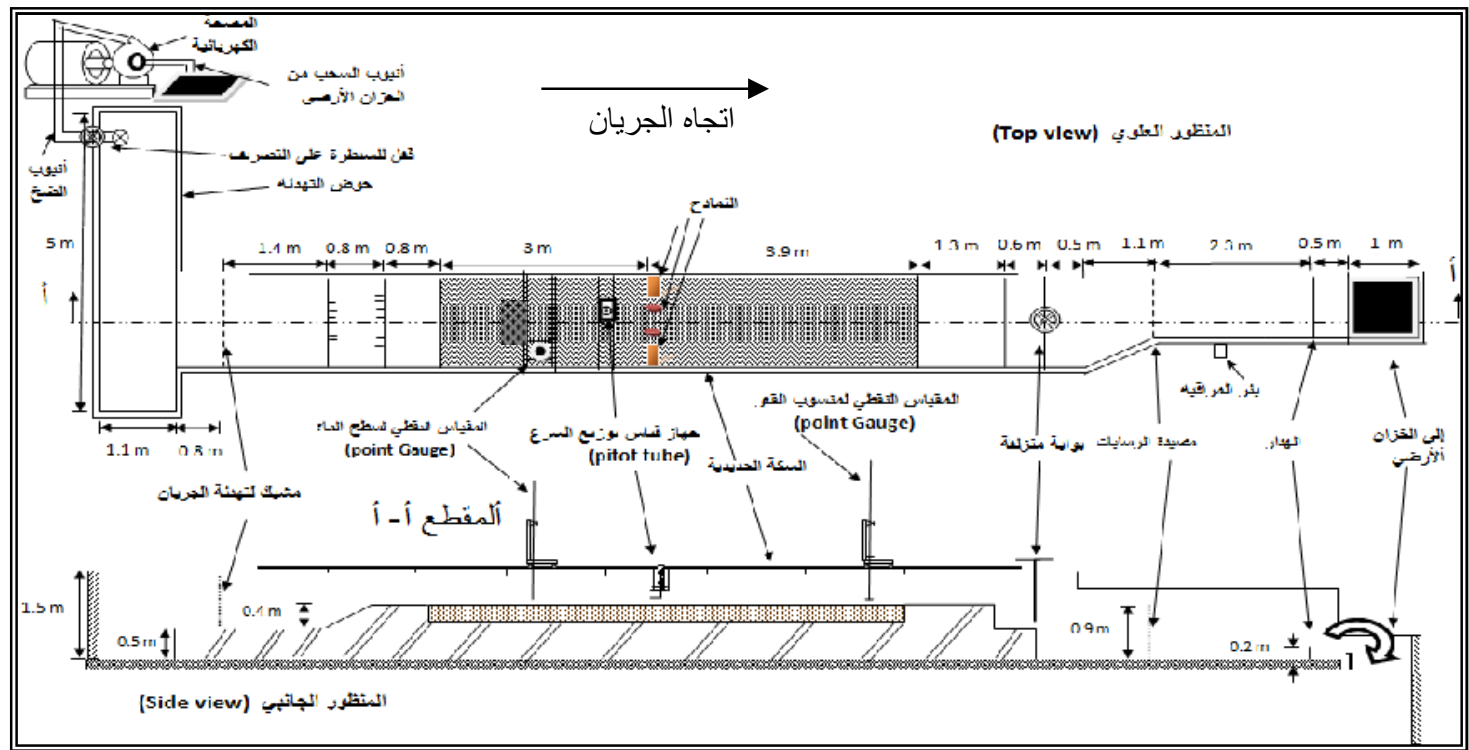

شكل رقم (1) القناة المختبرية المستخدمة في أداء التجارب

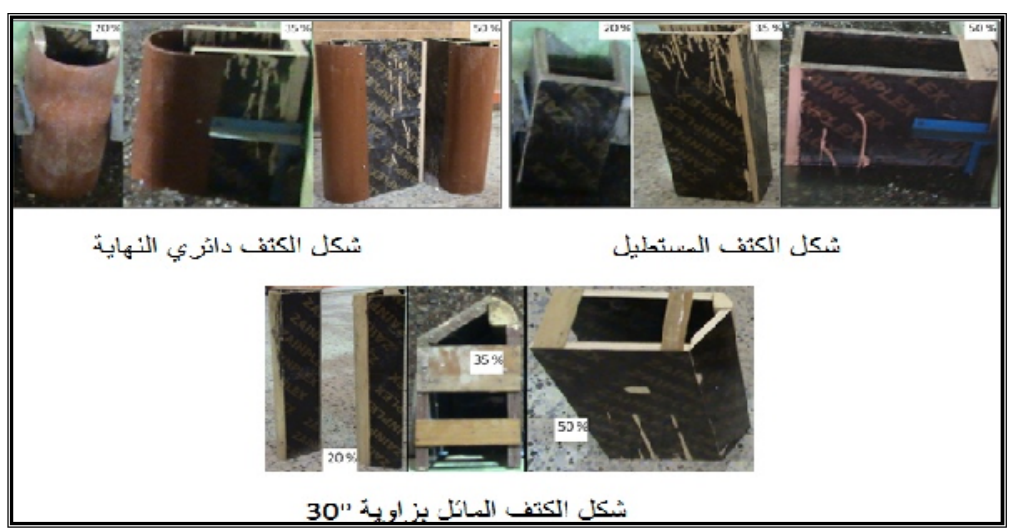

اللوحة رقم (1) الأكتاف المستخدمة في أداء التجارب

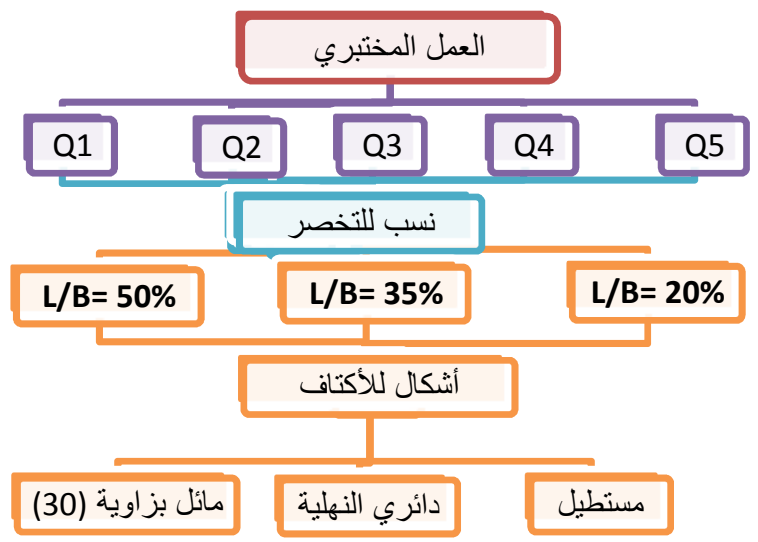

شكل رقم (2) جدول العمل ألمختبري. 


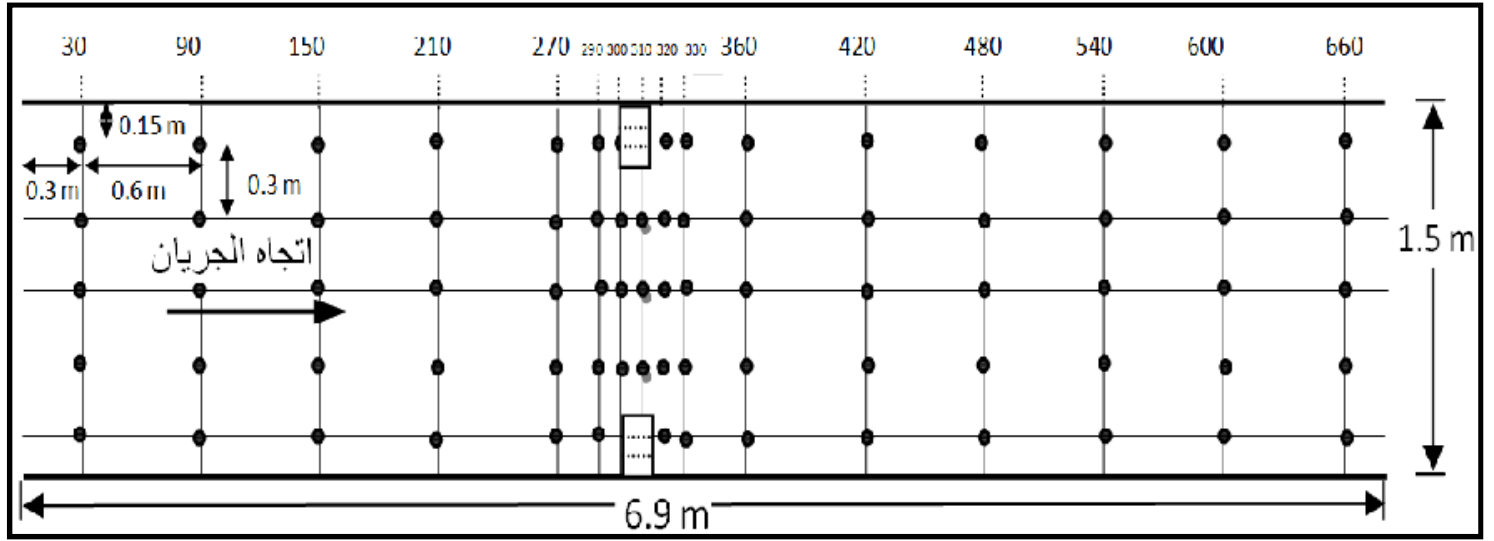

شكل رقم(3) التشبيك المتبع في أخذ مناسيب سطح الماء ومناسيب القعر في جميع التجارب

الجدول (1): القر اءات المختبرية لمنسوب سطح القعر بالـ(cm) للتصريف (60 L/s) ولثنكل الكتف الدائري الذي يحدث نسبة تخصر في مقطع القناة مقدار ها (20\%).

\begin{tabular}{|c|c|c|c|c|c|}
\hline \multicolumn{5}{|c|}{ الأبعاد من الضأة اليسرى للجريان(cm) } & \multirow{2}{*}{ التسافة من مركز } \\
\hline 135 & 105 & 75 & 45 & 15 & \\
\hline-0.1 & 0 & 0 & -0.1 & -0.3 & -280 \\
\hline-0.1 & 0.2 & 0 & -0.3 & -0.1 & -220 \\
\hline 0.1 & 0.3 & -0.1 & 0.2 & 0.0 & -160 \\
\hline 0.1 & 0.1 & 0.2 & 0 & -0.2 & -100 \\
\hline 0 & 0.1 & 0.1 & -0.1 & -0.3 & -40 \\
\hline 0.1 & 0.1 & -0.2 & -0.1 & -0.2 & -20 \\
\hline-4.7 & 0 & 0.1 & 0.1 & -4 & -10 \\
\hline-6.3 & 0.1 & -0.1 & 0 & -6.1 & 0 \\
\hline-3.2 & -0.1 & -0.4 & -0.1 & -3 & 10 \\
\hline 2.3 & -0.5 & -0.3 & -0.4 & 2.2 & 20 \\
\hline 2.2 & 0 & 0 & 0.2 & 2 & 50 \\
\hline 0.4 & 0.9 & 0.5 & 0 & -0.2 & 110 \\
\hline 0.1 & 0.4 & 0.1 & -0.3 & -0.7 & 170 \\
\hline 0 & 0.1 & -0.6 & -0.4 & -0.2 & 230 \\
\hline-0.1 & 0.1 & -0.5 & -0.4 & -0.6 & 290 \\
\hline-0.7 & -0.9 & -0.5 & -1 & -1.3 & 350 \\
\hline & 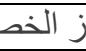 & كتف. & مصل & cm) & أعمق نحر \\
\hline
\end{tabular}

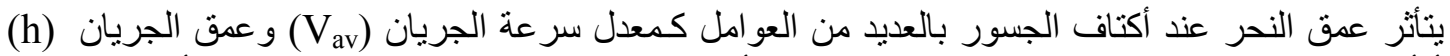

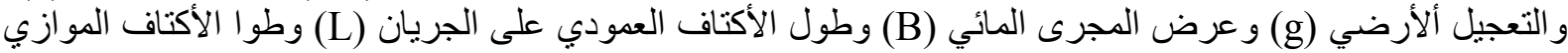

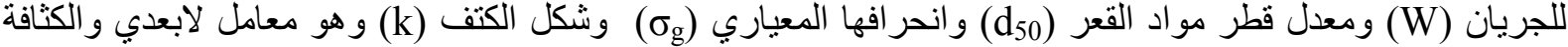

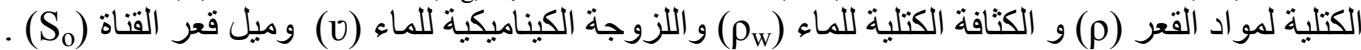

تم أجراء التحليل البعدي باستخدام نظرية باكينكهام (Buckingham theory) وكاءة) باعتبار أن المتغيرات ألأساسية هي عرض القناة (B) و التعجيل ألأرضي (g) وكثافة الماء (م)، وكاكنام (ولت نتيجة التحليل كالتالي:

$\frac{d_{s}}{(B h)^{0.5}}=f\left(F_{r}, \frac{L}{\mathrm{~B}}, \frac{\mathrm{W}}{\mathrm{B}}, \frac{\mathrm{h}}{\mathrm{d}_{50}}, \frac{\mathrm{B}}{\mathrm{d}_{50}}, \mathrm{~S}_{\mathrm{o}}, k, \frac{\rho_{w}}{\rho}, R e, \sigma_{g}\right)$ 


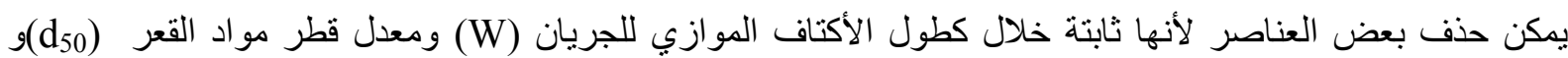

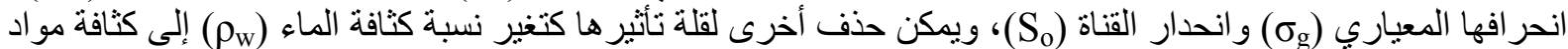

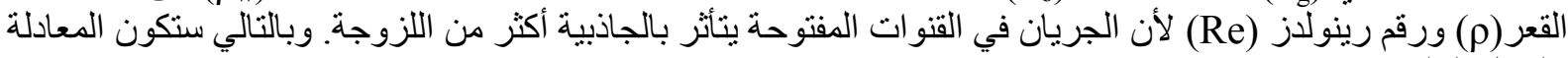

$\frac{d_{s}}{(B h)^{0.5}}=f\left(F_{r}, \frac{L}{\mathrm{~B}}, k\right)$ بالثكل التالي.

$\frac{d_{s}}{(B h)^{0.5}}=A F_{r}{ }^{\mathrm{n}}\left(\frac{L}{\mathrm{~B}}\right)^{m}$

تم تثبيت شكل الكتف لكل حالة أي أن (k) يمكن تثبيتها وصياغة المعادلة بالثكل النالي:

تؤخذ قيم (A ,n ,m) من الجدول التالي وحسب شكل الكتف.أذ نم إستتناج هذه القيم من بستخدام برنامج (SPSS-11.5) وبالأعتماد على بيانات التجارب المختبرية. جدول (2): قيم معاملات المعادلة (3) مع معامل التحديد لكل حالة

\begin{tabular}{|c|c|c|c|c|}
\hline قيمة معامل التحديد (R2) & A & $\mathrm{m}$ & $\mathrm{n}$ & شكل الكتف \\
\hline 0.9592 & 14.405 & 1.06 & 3.11 & المستطيل \\
\hline 0.871 & 13.974 & 1.101 & 3.298 & دائري النهاية \\
\hline 0.911 & 36.37 & 0.9097 & 4.564 & المائل بزاوية ( 30) \\
\hline
\end{tabular}

و لإيجاد معادلة شاملة لجميع الحالات تم أيجاد معامل الثكل (K) للكتف الدائري النهاية و المائل بزاوية (30) من تحلئل

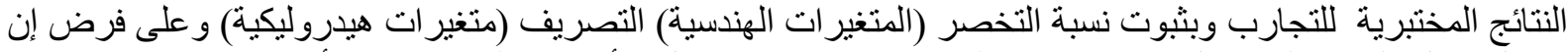

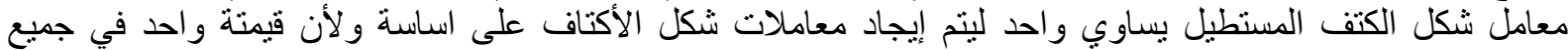

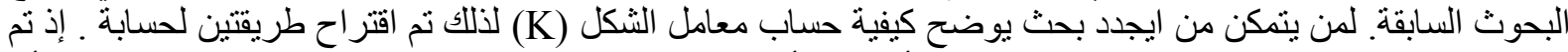

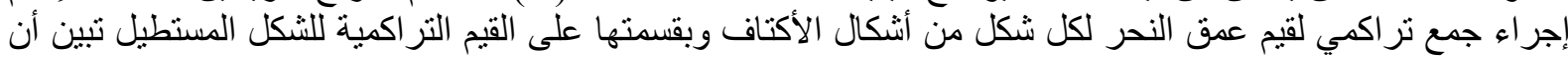

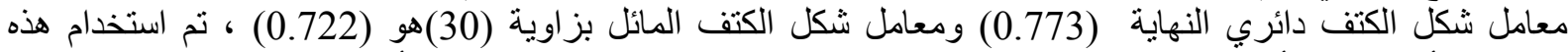

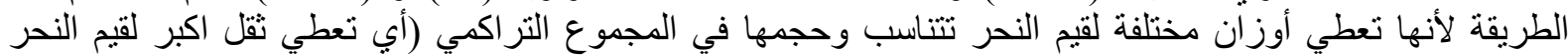

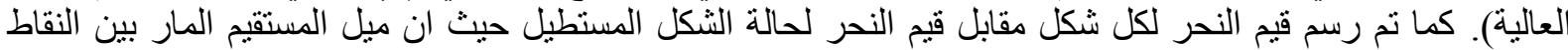

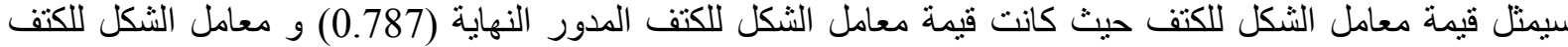

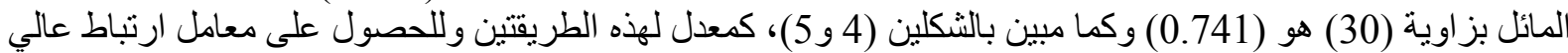

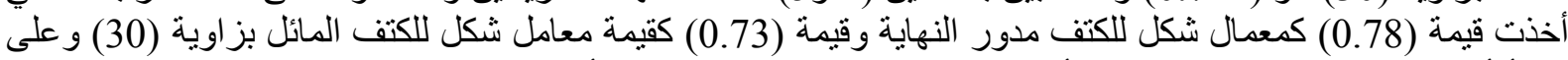

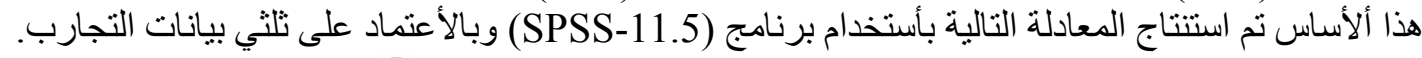
$\frac{d_{s}}{(B h)^{0.5}}=30.3 K F_{r}^{3.853}\left(\frac{L}{\mathrm{~B}}\right)^{1.115}$

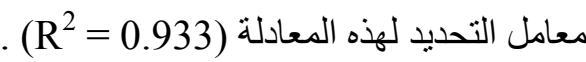

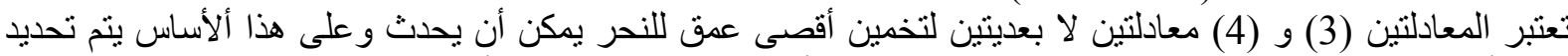

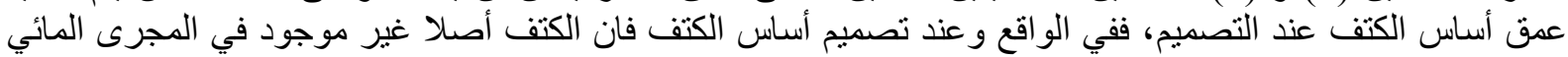

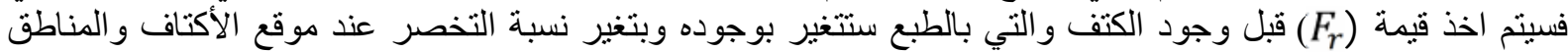

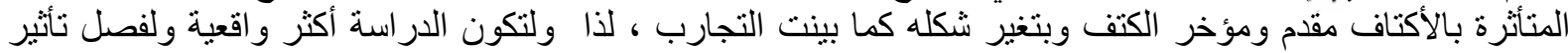

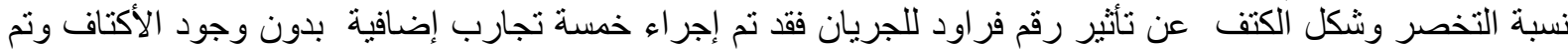

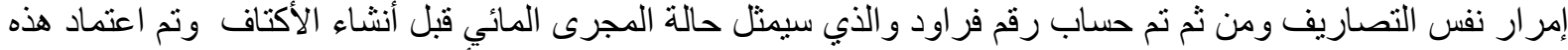

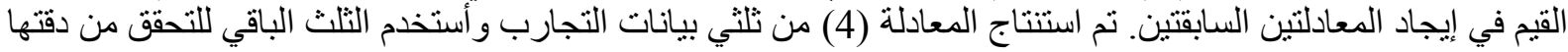

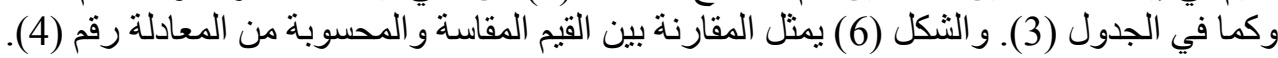


الجدول (3): ثلث بيانات التجارب للتحقق من دقة المعادلة (4).

\begin{tabular}{|c|c|c|c|c|c|c|c|c|c|c|}
\hline Err \% & $\begin{array}{l}\text { ds (cm) } \\
\text { المحسوبة }\end{array}$ & $\mathrm{k}$ & $\begin{array}{l}\text { المقاسة } \\
\text { المع (cm) }\end{array}$ & $\frac{d s}{(h * b)^{0.5}}$ & $\mathrm{Fr}$ & $\mathrm{v}_{\mathrm{a}}(\mathrm{m} / \mathrm{s})$ & $\begin{array}{c}\mathrm{Y} \\
(\mathrm{cm})\end{array}$ & $\begin{array}{l}\text { التصريف } \\
\text { L/S(Q) }\end{array}$ & $(1 / B)$ & شكتل \\
\hline 7.723 & 8.3971 & 1 & 9.1 & 0.28285 & 0.3558 & 0.2927 & 6.9 & 30.3 & 0.5 & \multirow{5}{*}{ 㝵 } \\
\hline 0.227 & 14.168 & 1 & 14.2 & 0.3860 & 0.3936 & 0.3703 & 9.02 & 50.1 & 0.5 & \\
\hline-5.763 & 9.519 & 1 & 9 & 0.2447 & 0.3936 & 0.3703 & 9.02 & 50.1 & 0.35 & \\
\hline 16.404 & 3.929 & 1 & 4.7 & 0.1352 & 0.3733 & 0.3319 & 8.06 & 40.13 & 0.2 & \\
\hline 14.544 & 6.666 & 1 & 7.8 & 0.2036 & 0.4176 & 0.4090 & 9.78 & 60 & 0.2 & \\
\hline 18.871 & 6.490 & 0.78 & 8 & 0.2487 & 0.3558 & 0.2928 & 6.9 & 30.3 & 0.5 & \multirow{5}{*}{$\begin{array}{l}\frac{1}{3} \\
y \\
\frac{7}{3} \\
\frac{7}{3}\end{array}$} \\
\hline-14.496 & 14.312 & 0.78 & 12.5 & 0.3264 & 0.4176 & 0.4090 & 9.78 & 60 & 0.5 & \\
\hline 4.452 & 7.357 & 0.78 & 7.7 & 0.2093 & 0.3936 & 0.3703 & 9.02 & 50.1 & 0.35 & \\
\hline 15.644 & 3.037 & 0.78 & 3.6 & 0.1035 & 0.3733 & 0.3319 & 8.06 & 40.13 & 0.2 & \\
\hline 18.223 & 5.152 & 0.78 & 6.3 & 0.1645 & 0.4176 & 0.4090 & 9.78 & 60 & 0.2 & \\
\hline 11.198 & 3.819 & 0.73 & 4.3 & 0.1465 & 0.3232 & 0.2425 & 5.74 & 20.88 & 0.5 & \multirow{5}{*}{$\begin{array}{l}\overline{3} \\
-3 \\
.7 \\
\overline{3} \\
\overline{3}\end{array}$} \\
\hline-15.961 & 7.885 & 0.73 & 6.8 & 0.1956 & 0.3733 & 0.3319 & 8.06 & 40.13 & 0.5 & \\
\hline 0.041 & 5.298 & 0.73 & 5.3 & 0.1524 & 0.3733 & 0.3319 & 8.06 & 40.13 & 0.35 & \\
\hline 19.096 & 6.877 & 0.73 & 8.5 & 0.2311 & 0.3936 & 0.3703 & 9.02 & 50.1 & 0.35 & \\
\hline-23.415 & 2.839 & 0.73 & 2.3 & 0.0661 & 0.3733 & 0.3319 & 8.06 & 40.13 & 0.2 & \\
\hline
\end{tabular}

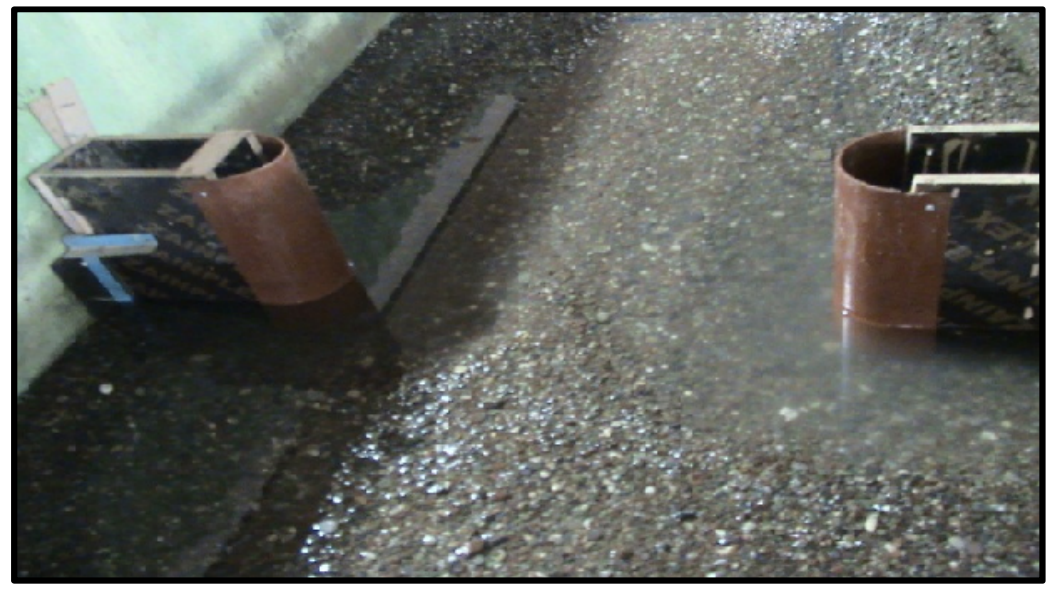

اللوحة (2): شكل وموقع حفرة النحر للكتف دائري النهاية ولنسبة تخصر (50\%).

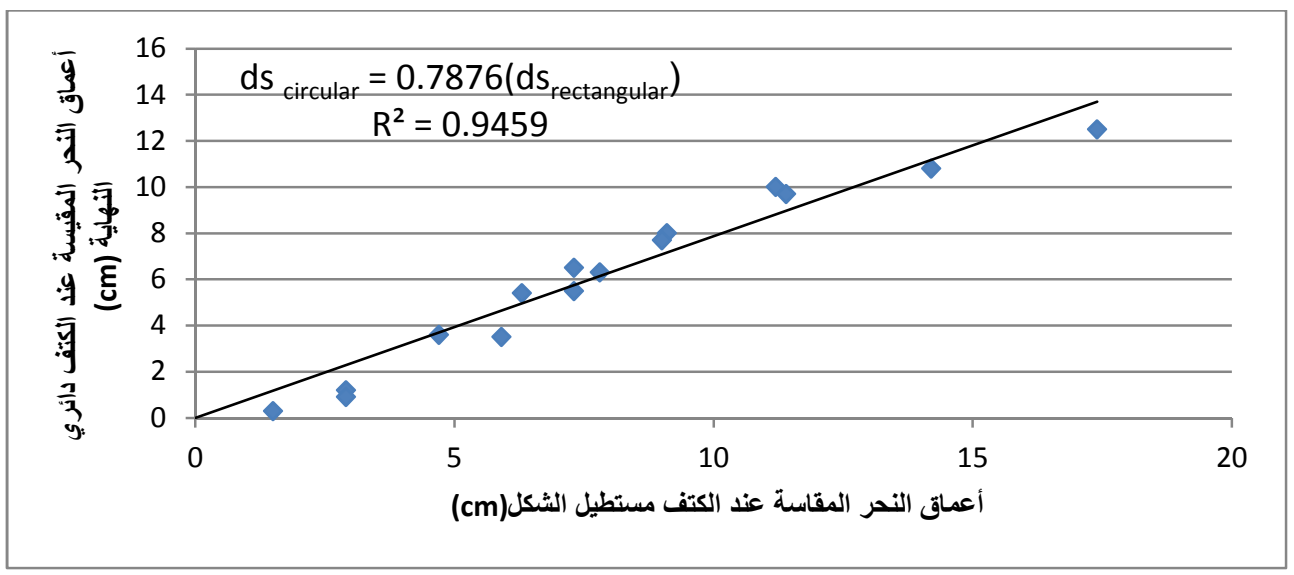

شكل رقم (4): علاقة عمق النحر لحالة الكتف دائري النهاية مع الكثف المستطيل 
محمد: تأثير نسبة التخصر وشكل الكتف على عمق النحر الموضعي عند أكتاف الجسور

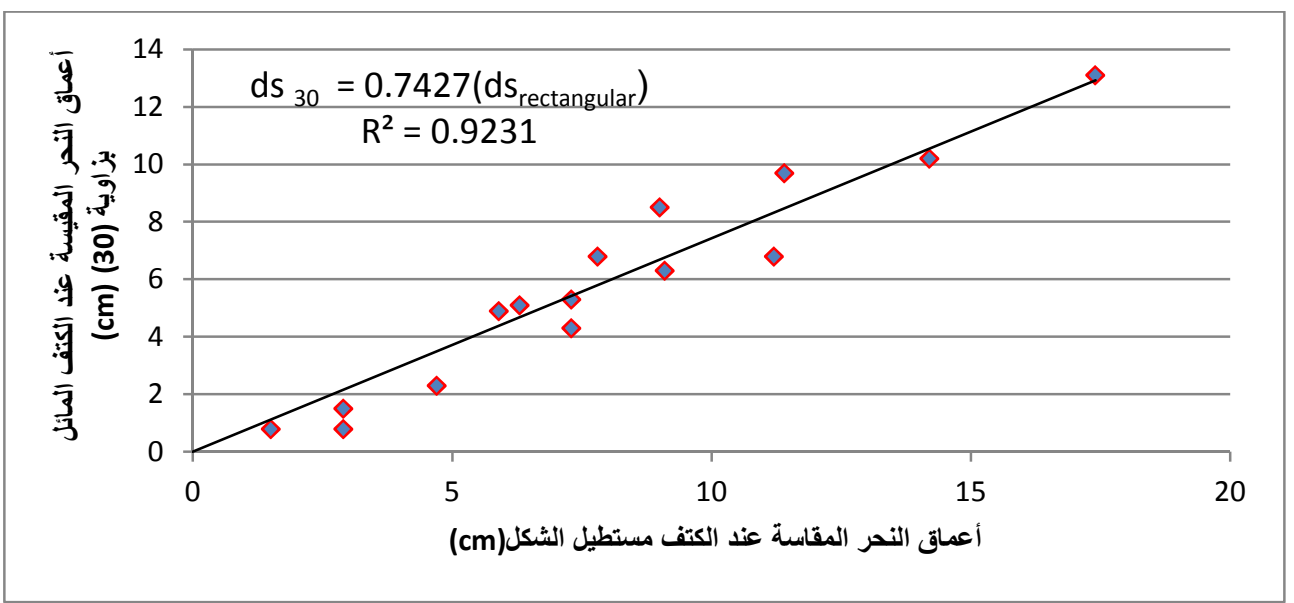

شكل رقم (5): علاقة عمق النحر لحالة الكتف المائل بز اوية (30) مع الكتف المستطيل

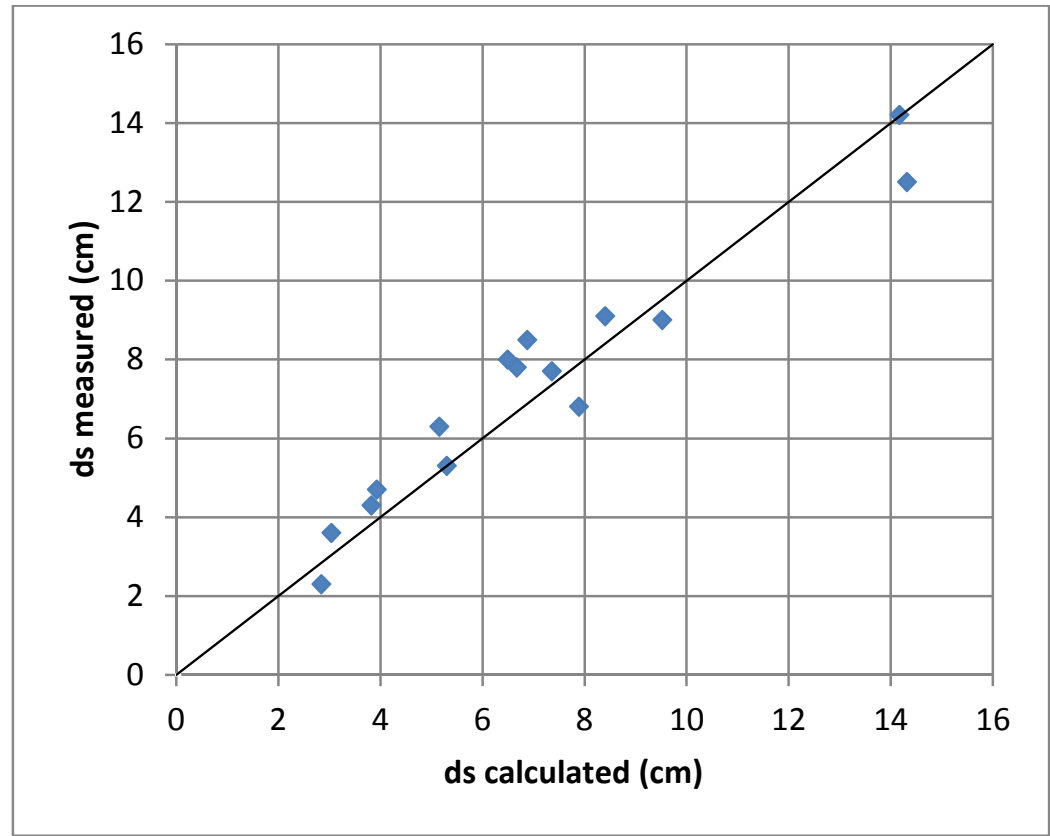

الثكل(6): مقارنة بين القيم المقاسة و المحسوبة من المعادلة (4).

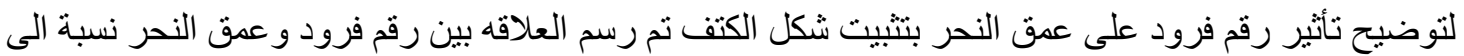

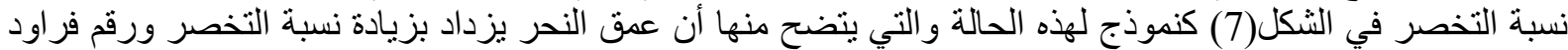

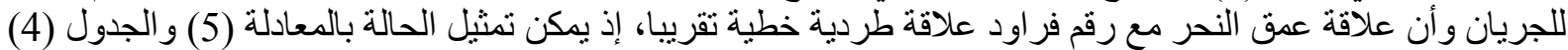

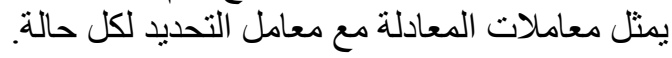

$\frac{d s}{(b h)^{0.5}}=a(f r)+b$ 


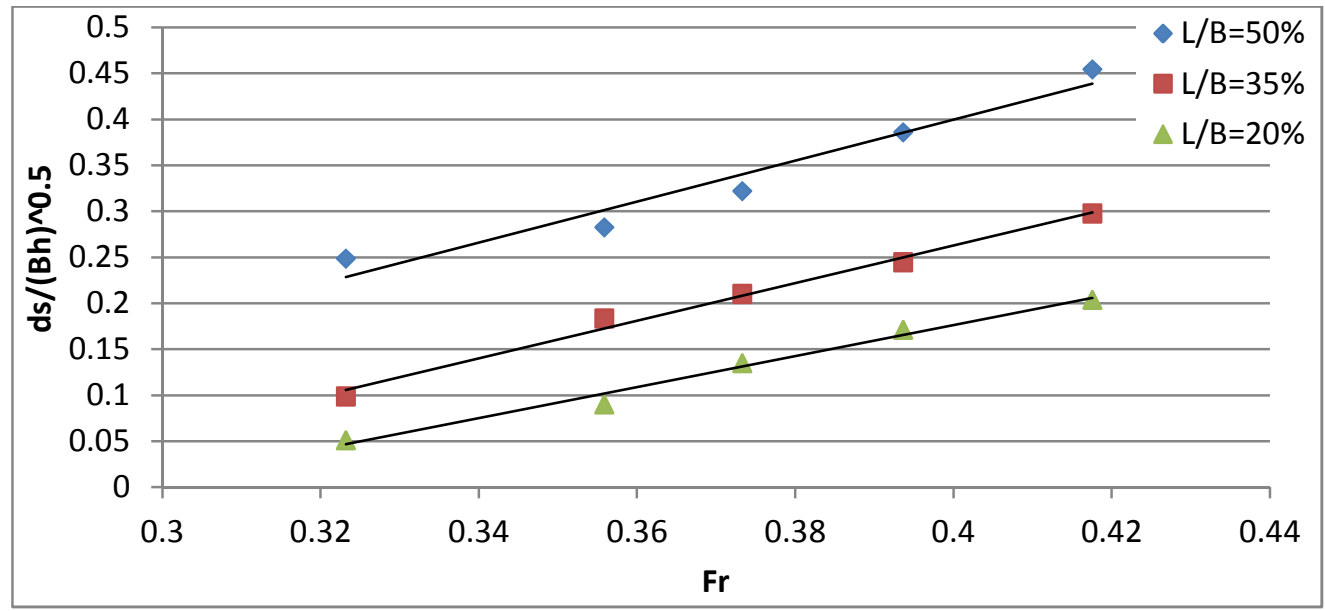

الثكل(7): علاقة عمق النحر اللابعدي مع رقم فر اود ولثلاث نسب للتخصر لحالة الكتف المستطيل.

الجدول (4):قيم معاملات المعادلة (5).

\begin{tabular}{|c|c|c|c|c|}
\hline معامل التحديد (R) & $\mathrm{b}$ & $\mathrm{a}$ & نسبة التخصر (L/B) & شكل الكتق \\
\hline 0.9514 & -0.4917 & 2.2284 & $50 \%$ & \multirow{3}{*}{ 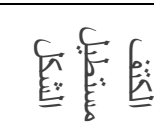 } \\
\hline 0.991 & -0.5554 & 2.0453 & $35 \%$ & \\
\hline 0.9861 & -0.4983 & 1.6864 & $20 \%$ & \\
\hline 0.9541 & -0.2687 & 1.442 & $50 \%$ & \multirow{3}{*}{ 点: } \\
\hline 0.9674 & -0.7017 & 2.3188 & $35 \%$ & \\
\hline 0.9144 & -0.5944 & 1.8379 & $20 \%$ & \\
\hline 0.9167 & -0.5375 & 2.0633 & $50 \%$ & \multirow{3}{*}{ 可 } \\
\hline 0.9384 & -0.7254 & 2.3843 & $35 \%$ & \\
\hline 0.8742 & -0.5607 & 1.7409 & $20 \%$ & \\
\hline
\end{tabular}

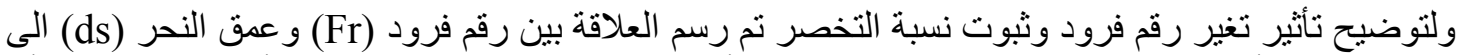

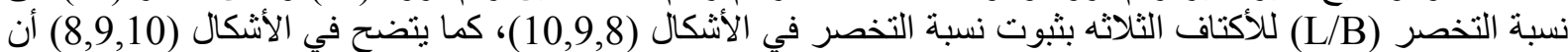

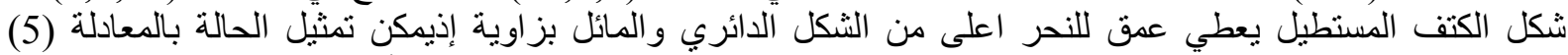

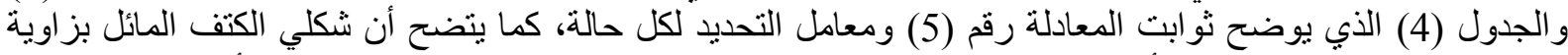

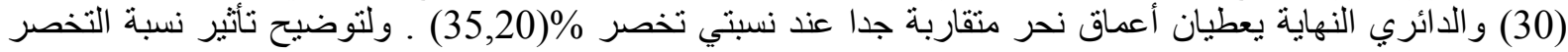

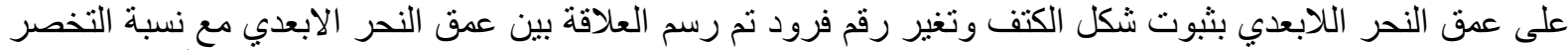

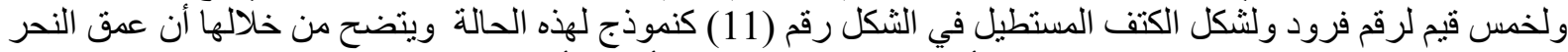

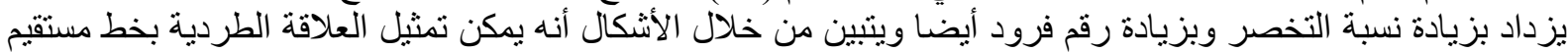

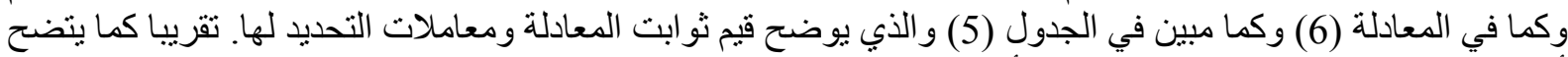

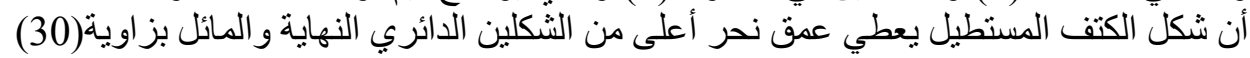

$\frac{d s}{(b h)^{0.5}}=a\left(\frac{L}{B}\right)+b$ 
محمد: تأثير نسبة التخصر وشكل الكتف على عمق النحر الموضعي عند أكتاف الجسور

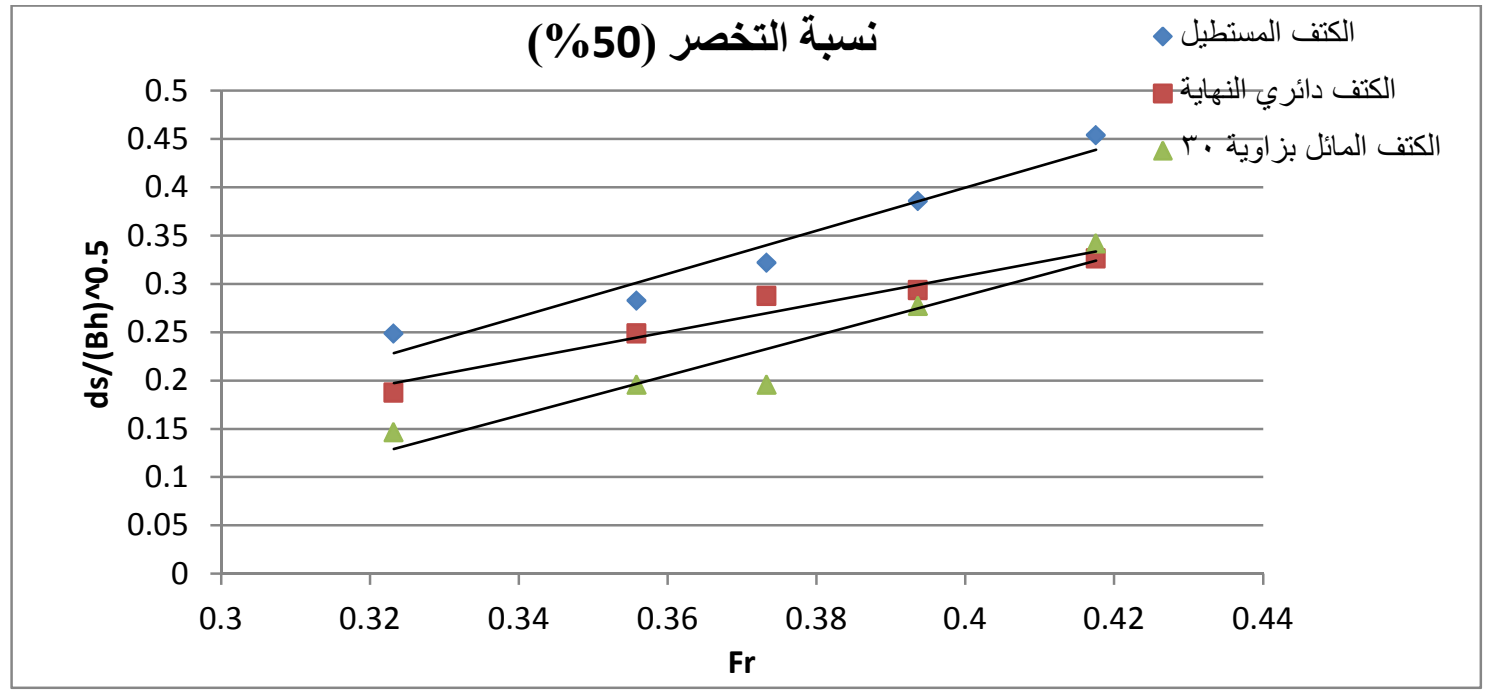

الثكل رقم (8) علاقة عمق النحر اللابعدي مع رقم فراود و لأشكال الأكتاف الثلاث بثبوت نسبة التخصر.

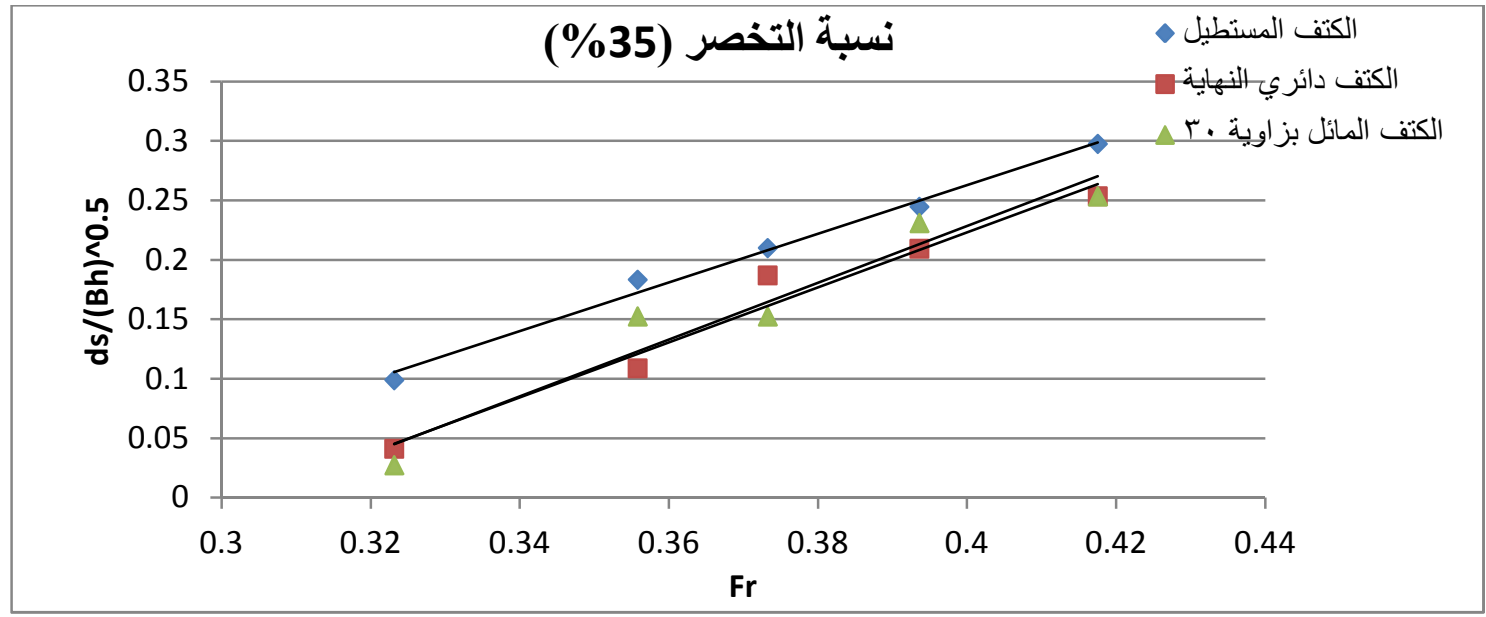

الثكل رقم (9) علاقة النحر اللابعدي مع رقم فر اود و لأشكال الأكتاف الثلاث بثبوت نسبة التخصر.

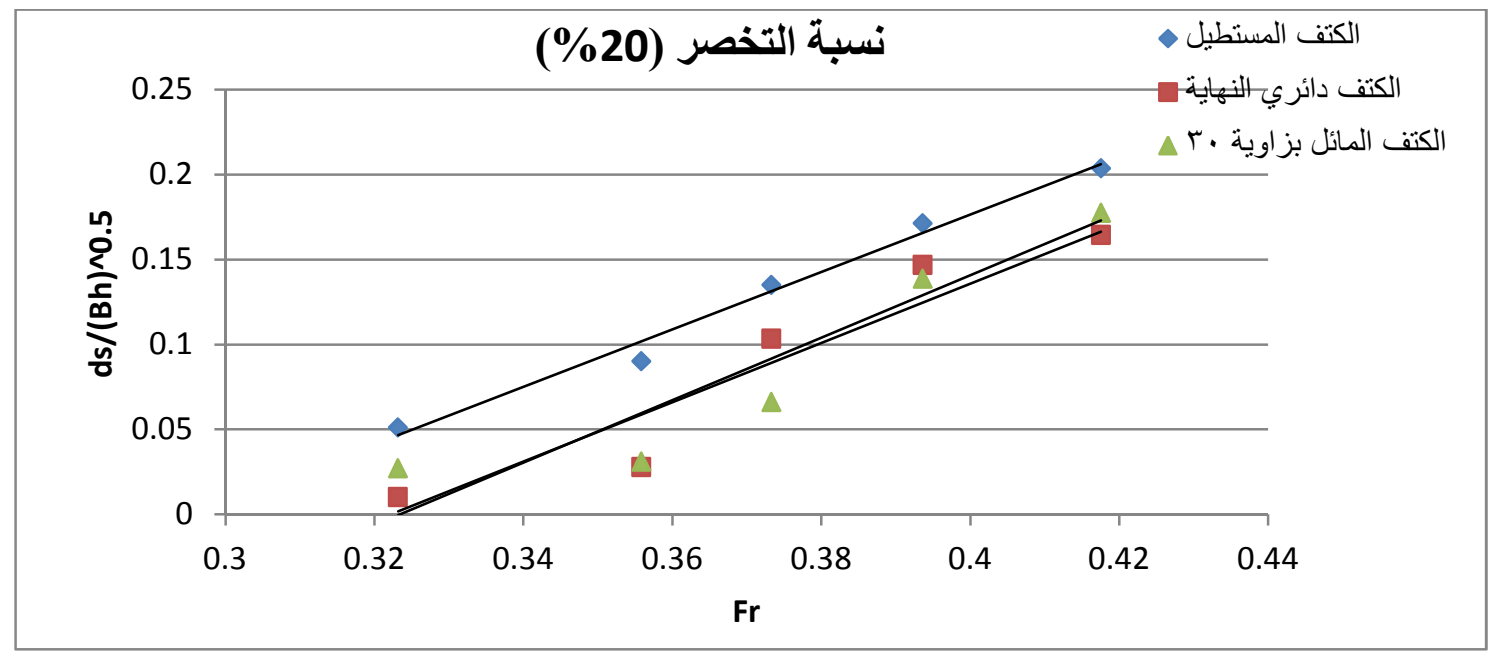

الثكل رقم (10) علاقة عمق النحر اللابعدي مع رقم فر اود و لأشكال الأكتاف الثناث بثبوت نسبة التخصر. 


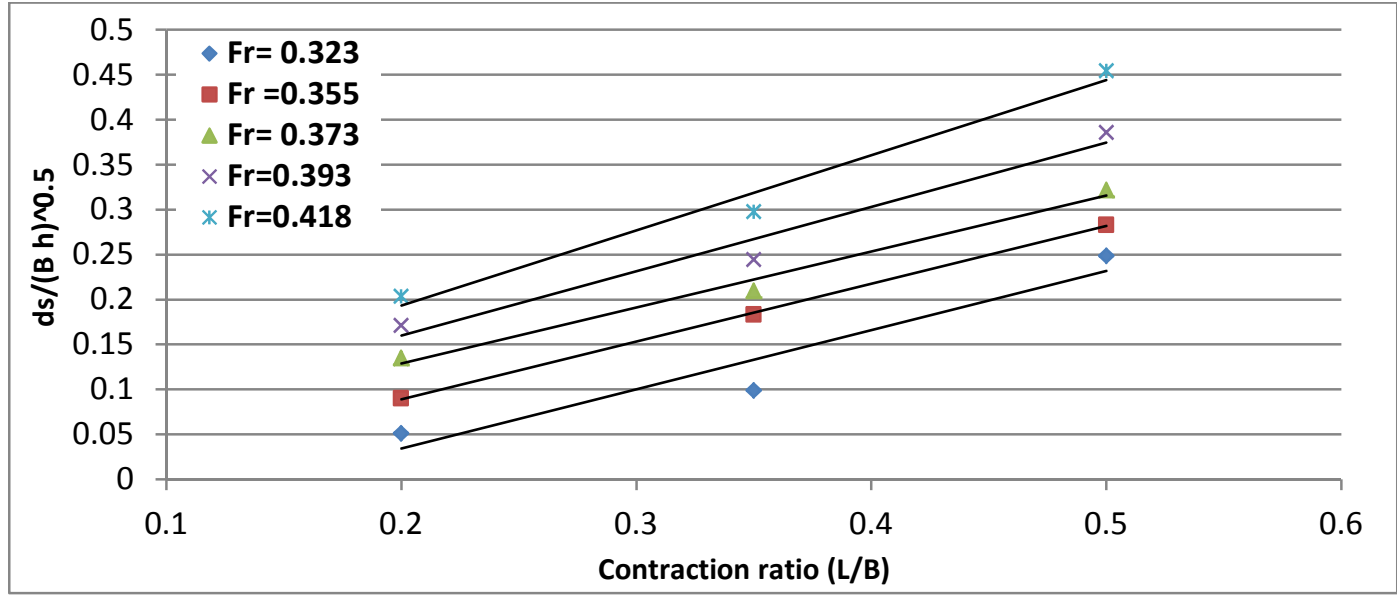

الثكل (11). علاقة عمق النحر اللابعدي مع نسبة التخصر ولخمس قيم لفراود ولنكل الكتف المستطيل. الجدول (5): ثو ابت المعادلة رقم (6) ومعاملات التحديد.

\begin{tabular}{|c|c|c|c|c|}
\hline معامل التحديد (R/2) & b & $\mathrm{a}$ & $\mathrm{Fr}$ & شكل الكتف \\
\hline 0.9181 & -0.0977 & 0.6589 & 0.3232 & \multirow{5}{*}{ 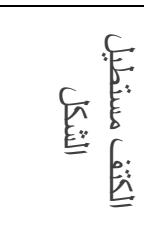 } \\
\hline 0.9997 & -0.0394 & 0.6424 & 0.3558 & \\
\hline 0.9868 & 0.0043 & 0.6231 & 0.3733 & \\
\hline 0.9677 & 0.0168 & 0.7159 & 0.3936 & \\
\hline 0.9796 & 0.0261 & 0.8355 & 0.4176 & \\
\hline 0.8753 & -0.1272 & 0.5907 & 0.3232 & \multirow{5}{*}{ 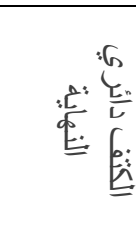 } \\
\hline 0.9767 & -0.129 & 0.7356 & 0.3558 & \\
\hline 0.9971 & -0.022 & 0.6135 & 0.3733 & \\
\hline 0.9927 & 0.0453 & 0.4894 & 0.3936 & \\
\hline 0.9969 & 0.0592 & 0.5396 & 0.4176 & \\
\hline 0.75 & -0.0721 & 0.3976 & 0.3232 & \multirow{5}{*}{ ف) } \\
\hline 0.931 & -0.0658 & 0.5491 & 0.3558 & \\
\hline 0.9643 & -0.0129 & 0.4314 & 0.3733 & \\
\hline 0.9643 & 0.0539 & 0.4622 & 0.3936 & \\
\hline 0.9979 & 0.0657 & 0.5483 & 0.4176 & \\
\hline
\end{tabular}

1- تبين من تحليل النتائج المختبرية أن علاقة عمق النحر مع كل من نسبة التخصر ورقم فرود للجريان علاقه طردية

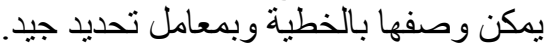

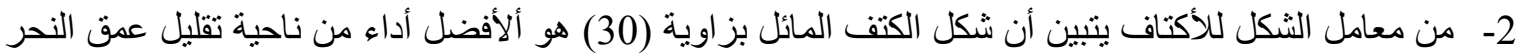

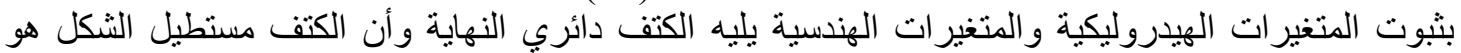

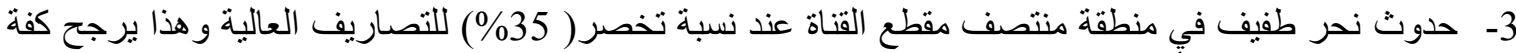

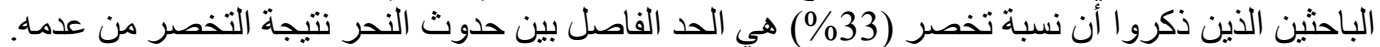

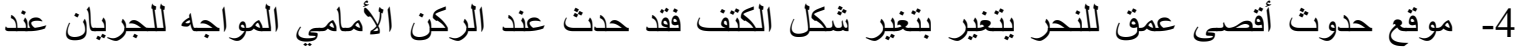

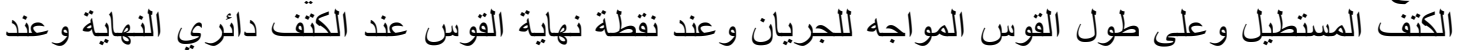

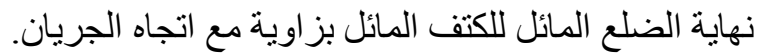

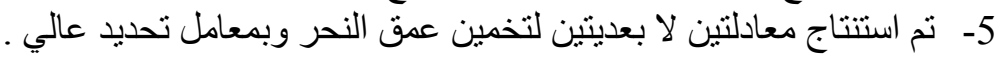




\section{References:}

1- Ballio, F., Teruzzi, A. and Radice, A. (2009), " Constriction Effects in Clear-Water Scour at Abutments", ASCE, J. of Hydraulic Engineering, Vol. 135, No. 2, pp. 140-145.

2- Cardoso, A. H., and Bettess, R. 1999, "Effects of time and channel geometry on scour at bridge abutments", ASCE, J. Hydraul. Eng., VOL, 125. NO, 4. PP, 388-399.

3- Coleman, S. E., Lauchlan, C. S., and Melville, B. W. 2003. "Clear-water scour development at bridge abutments." ASCE J. Hydraul. Res. VOL,41.NO,5. PP, 521-531.

4- Fael, C. M. S., Simarro-Grande, G., Martìn-Vide, J. P., and Cardoso, A.H. 2006. "Local scour at vertical-wall abutments under clear-water flow conditions", ASCE, Water Resour. Res., VOL,42. W10408.

5- Oliveto, G., and Hager, W. H. 2002. "Temporal evolution of clear-water pier and abutment scour", ASCE, J. Hydraul. Eng., VOL,128. NO,9. PP, 811-820.

6- Melville, B. W. 1992_. "Local scour at bridge abutments ."ASCE, J. Hydraul. Eng., VOL,118.NO,4. PP, 615-631

7- Yousefpour, N., Jahedkar, K.,and Arjwech, R., (2011), " Determination of Unknown Foundation of Bridges for Scour Evaluation Using Artificial Neural Networks" ASCE, Geo-Frontiers.

$$
\text { تم اجراء البحث في كلية ألهندة = جامعة ألموصل }
$$

